\title{
TNFAIP8 promotes cisplatin resistance in cervical carcinoma cells by inhibiting cellular apoptosis
}

\author{
SUXIA WU ${ }^{1,2}$, WEIHUA $\mathrm{LI}^{3}$, ZHENGHUI WU ${ }^{1,4}$, TIANRAN CHENG ${ }^{1}$, PING WANG $^{1,4}$, NA LI $^{1,4}$, \\ XIAONAN LIANG ${ }^{1,4}$, MENGMENG CHI ${ }^{1}$, SHUMAN ZHANG ${ }^{5}$, YUANFANG MA ${ }^{1,4}$, \\ YANYUN $\mathrm{LI}^{5}$ and LIHUI CHAI ${ }^{1,4}$ \\ ${ }^{1}$ Joint National Laboratory for Antibody Drug Engineering, Henan University; \\ ${ }^{2}$ Department of Pathology, Henan University School of Basic Medical Sciences, Kaifeng, Henan 475004; \\ ${ }^{3}$ Clinical Laboratory, The First Affiliated Hospital of Henan University, Kaifeng, Henan 475001; \\ ${ }^{4}$ Department of Immunology, Henan University School of Basic Medical Sciences, Kaifeng, Henan 475004; \\ ${ }^{5}$ Department of Gynaecology and Obstetrics, Affiliated Huaihe Hospital of Henan University, \\ Kaifeng, Henan 475000, P.R. China
}

Received May 19, 2018; Accepted January 21, 2019

DOI: $10.3892 / \mathrm{ol} .2019 .10076$

\begin{abstract}
Cervical cancer is the second most prevalent malignant tumor in women worldwide. Failure of successful treatment is most prevalent in patients with the metastatic disease and the chemotherapy refractory disease. Tumor necrosis factor $\alpha$-induced protein 8 (TNFAIP8) serves as an anti-apoptotic and pro-oncogenic protein, and is associated with cancer progression and poor prognosis in a number of different cancer types. However, the physiological and pathophysiological roles of TNFAIP8 in cervical carcinogenesis and development remain poorly understood. In the present study, it was demonstrated that TNFAIP8 protein expression levels were significantly increased in cervical cancer tissues compared with the non-tumor adjacent tissues using immunohistochemistry. Additionally, it was demonstrated that TNFAIP8 overexpression is associated with cisplatin resistance. Furthermore, depletion of TNFAIP8 impaired HeLa cell proliferation and viability in vitro, improved cisplatin sensitivity, and promoted cisplatin-induced cellular apoptosis and death. Subsequent mechanistic analysis demonstrated that
\end{abstract}

Correspondence to: Dr Lihui Chai, Joint National Laboratory for Antibody Drug Engineering, Henan University, North Jinming Avenue, Kaifeng, Henan 475004, P.R. China

E-mail: clh0301@henu.edu.cn

Dr Yanyun Li, Department of Gynaecology and Obstetrics, Affiliated Huaihe Hospital of Henan University, 8 Baobei Road, Kaifeng, Henan 475000, P.R. China

E-mail: 13592146916@139.com

Abbreviations: TNFAIP8, tumor necrosis factor $\alpha$-induced protein 8; MAPK, mitogen-activated protein kinase; CDDP, Cis-diamminedichloroplatinum

Key words: TNFAIP8, cervical cancer, cisplatin, MAPK, apoptosis
TNFAIP8 silencing promoted caspase-8/-3 activation and p38 phosphorylation in HeLa cells treated with cisplatin, whereas apoptosis regulator B-cell lymphoma-2 expression was inhibited with TNFAIP8-silenced HeLa cells following treatment with cisplatin. These data suggested that TNFAIP8 serves as an anti-apoptotic protein against cisplatin-induced cell death, which eventually leads to chemotherapeutic drug-treatment failure. Therefore, the present data suggested that TNFAIP8 may be a promising therapeutic target for the treatment of cervical cancer.

\section{Introduction}

Cervical cancer is the second most prevalent malignant tumor in women worldwide and the fourth leading cause of cancer-associated mortalities among women $(1,2)$. Surgery, chemotherapy and radiotherapy are the standard treatment options for patients with cervical cancer; however, the 5 year survival rate for advanced and recurrent cervical cancer is only 10-20\%; with metastasis and chemotherapeutic agent resistance being the principal causes of treatment failure (3). Cis-diamminedichloroplatinum (II) (more commonly referred to as cisplatin) is a small-molecular platinum-based compound that appears to be the most effective anti-tumor agent in patients with advanced and recurrent cervical cancer (4). However, either intrinsic or acquired resistance to cisplatin may develop, seriously compromising the efficacy of cisplatin (5). Previous studies investigating the mechanisms of cisplatin resistance have identified a number of complex resistance mechanisms $(6,7)$. As a natural defense mechanism developed to protect cells from environmental toxins, cisplatin resistance requires numerous epigenetic and genetic alterations that support cell survival, including cell growth, apoptosis, developmental pathways, DNA damage repair and cellular accumulation of cisplatin (8).

Tumor necrosis factor $\alpha$-induced protein 8 (TNFAIP8 or TIPE; additionally termed SCC-S2, NDED or GG2-1) serves 
as an anti-apoptotic and pro-oncogenic signaling molecule (9-12). A previous study suggested that tumor necrosis factor $\alpha$ (TNF- $\alpha)$ stimulation and the activation of the nuclear factor- $\kappa \mathrm{B}$ pathway may upregulate the mRNA expression levels of TNFAIP8 in head and neck squamous cell carcinoma cell lines (13). In addition, TNFAIP8 overexpression was associated with cancer progression and poor prognosis in a number of different cancer types (12,14-18). Silencing TNFAIP8 in a variety of cells promoted cellular apoptosis, attenuated cellular proliferation and the production of matrix metalloproteinase (MMP)-1 and MMP-9 (19). However, the physiological and pathophysiological roles of TNFAIP8 in cervical carcinogenesis and development remain poorly understood. It is unclear how the expression of TNFAIP8 in cervical cancer promotes cisplatin resistance.

In the present study, TNFAIP8 expression in cervical cancer tissues compared with matching adjacent tissues was examined and it was demonstrated that increased TNFAIP8 was associated with cisplatin resistance. Additionally, the effect of TNFAIP8 inhibition using short hairpin (sh)RNA on cisplatin-induced cellular apoptosis in vitro was examined. Subsequent examination of the potential underlying mechanisms suggested that TNFAIP8 promoted cisplatin resistance by inhibiting cellular apoptosis.

\section{Materials and methods}

Patient tissue samples. In total, 40 pairs of cervical cancer tissues in addition to the corresponding adjacent tissues were collected from 40 female patients (39-67 years old) who underwent surgical resection at the Affiliated Huaihe Hospital of Henan University (Kaifeng, China) between April 2012 and December 2012. These patients were pathologically diagnosed with cervical cancer by two pathologists. All patients had no metastatic tumors, no serious complications and no other malignant tumors. Prior to cervical resection, none of the patients had received radiotherapy or chemotherapy. All patients received cisplatin-based chemotherapy following surgery. Patients were identified as cisplatin-sensitive if no neoplasm was found by imaging within 12 months of chemotherapy, or as cisplatin-resistant if neoplasm was found. The Committee for Ethical Review at Henan University School of Medicine (Kaifeng, China) approved the protocol, and written informed consent was provided by all patients.

Immunohistochemistry. Cervical cancer specimens and adjacent tissues were fixed with $4 \%$ paraformaldehyde overnight at room temperature, and embedded in paraffin and sectioned at a thickness of $4 \mu \mathrm{m}$ in the Department of Pathology, Affiliated Huaihe Hospital of Henan University. All sectioning was performed using standardized methods. Sections were deparaffinized in xylene twice for $10 \mathrm{~min}$, rehydrated in gradient ethanol (100, 90, 80, 70 and 60\%) once for $2 \mathrm{~min}$ at room temperature and subjected to heat-induced antigen retrieval and elimination of endogenous peroxidases by boiling in a water bath for $10 \mathrm{~min}$. Subsequently, sections were blocked with $10 \%$ goat serum (Wuhan Boster Biological Technology, Ltd., Wuhan, China) at room temperature for 15 min to prevent non-specific adsorption and incubated with a primary antibody against TNFAIP8 (1:100; ab195810; Abcam, Cambridge, MA,
USA) at $4^{\circ} \mathrm{C}$ overnight. Sections were subsequently incubated with a horseradish peroxidase-conjugated secondary goat anti-rabbit antibody (1:500; BA1056; Wuhan Boster Biological Technology, Ltd.) for $1.5 \mathrm{~h}$ at room temperature. Subsequently, the samples were stained with diaminobenzidine for $5 \mathrm{~min}$ and counterstained with hematoxylin (Beyotime Institute of Biotechnology, Haimen, China) for $2 \mathrm{~min}$ at room temperature, and sealed with neutral gum. TNFAIP8 staining was assessed as previously described (20) with specific modifications. All slides were independently analyzed with a light microscope (magnification $\mathrm{x} 100$ ) by two pathologists in a blinded manner and scored based on staining intensity as follows: i) 0 , No staining; ii) 1, weak staining; iii) 2 , moderate staining; and iv) 3 , strong staining. If there were discrepancies between the two pathologists, the final decision was made by another pathologist.

Cell culture. The human cervical cancer cell line (HeLa) was purchased from The Cell Bank of Type Culture Collection of Chinese Academy of Sciences (Shanghai, China). HeLa cells were cultured in Dulbecco's modified Eagle's medium (DMEM) (Gibco; Thermo Fisher Scientific, Inc., Waltham, MA, USA) containing certified $10 \%$ fetal bovine serum (Gibco; Thermo Fisher Scientific, Inc.), $100 \mathrm{U} / \mathrm{ml}$ penicillin and $100 \mu \mathrm{g} / \mathrm{ml}$ streptomycin (Beijing Solarbio Science \& Technology Co., Ltd., Beijing, China). A TNFAIP8-silenced HeLa cell line was established using lentiviral transfection using a pGLV-U6-Puro vector carrying TNFAIP8 shRNA (Shanghai GenePharma Co., Ltd., Shanghai, China). Briefly, infectious lentiviral vectors were harvested form HEK293T cells co-transfected with the recombinant competent virions (pGLV-U6-shTNFAIP8) and helper plasmids (pGag/Pol, pRev and pVSV-G) using Lipofectamine ${ }^{\circledR} 2000$ (Invitrogen; Thermo Fisher Scientific, Inc.) according to the manufacturer's protocol. HeLa cells were transfected with $10^{9}$ transducing units $/ \mathrm{ml}$ of lentiviruses in fresh transduction medium supplemented with $8 \mu \mathrm{g} / \mathrm{ml}$ Polybrene (Sigma-Aldrich; Merck KGaA, Darmstadt, Germany). Cells were cultured in complete medium containing puromycin $(2 \mu \mathrm{g} / \mathrm{ml})$ for at least 2 weeks prior to being used for experiments. TNFAIP8 expression was determined using both RT-qPCR and western blotting post-transduction. All cells were cultured in a humidified incubator containing $5 \%$ $\mathrm{CO}_{2}$ in compressed air at $37^{\circ} \mathrm{C}$.

Reverse transcription quantitative polymerase chain reaction $(R T-q P C R)$. Total RNA was extracted from HeLa cells using a RNAiso Plus extraction kit and reverse-transcribed into cDNA at $42^{\circ} \mathrm{C}$ for $1 \mathrm{~h}$ using the PrimeScript RT reagent kit (Takara Biotechnology Co., Ltd., Dalian, China). qPCR was performed using GoTaq qPCR Master Mix (Promega Corporation, Madison, WI, USA). For detection of TNFAIP8 mRNA expression levels, GAPDH was amplified in parallel as an internal control. The following primers were used for qPCR: TNFAIP8 forward, 5'-TCCATCGCC ACCACCTTA-3' and reverse, 5'-CTCTGCCTCCTTCTT GTTTT-3'; GAPDH forward, 5'-GGCAAATTCAACGGC ACAGTCA-3' and reverse, 5'-GTCTCGCTCCTGGAAGAT GGTGAT-3'. The qPCR conditions were denaturation at $95^{\circ} \mathrm{C}$ for $10 \mathrm{~min}$, followed by 40 cycles of denaturation at $95^{\circ} \mathrm{C}$ for $15 \mathrm{sec}$, annealing at $60^{\circ} \mathrm{C}$ for $30 \mathrm{sec}$ and extension at $55^{\circ} \mathrm{C}$ 
A
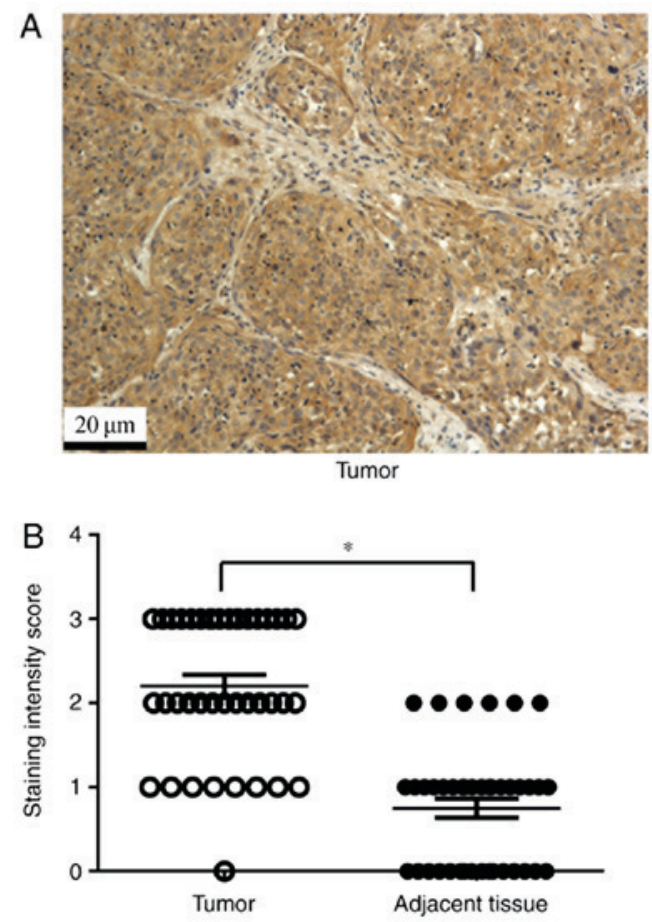
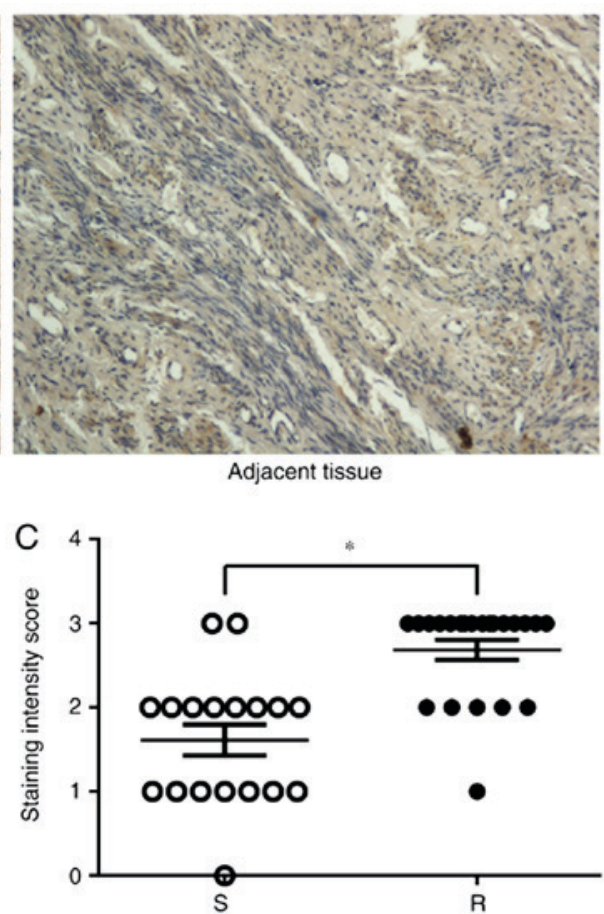

Figure 1. Expression of TNFAIP8 in cervical cancer tissues is associated with cisplatin resistance. (A) Immunohistochemical staining of TNFAIP8 expression in cervical cancer and adjacent non-tumor tissues. Brown staining denotes a positive signal. (B) Staining intensity scores for TNFAIP8 expression in cervical cancer and adjacent non-tumor tissues. (C) Staining intensity scores of TNFAIP8 expression in cervical cancer tissues from cisplatin-sensitive or cisplatin-resistant patients. Magnification, $\mathrm{x} 100$. ${ }^{*} \mathrm{P}<0.05$. S, sensitive to cisplatin; $\mathrm{R}$, resistant to cisplatin; TNFAIP8, tumor necrosis factor $\alpha$-induced protein 8 .

for $1 \mathrm{~min}$. The gene expression level was calculated using the $2^{-\Delta \Delta \mathrm{Cq}}$ method (21). The expression level in control cells was considered as 1.

Plate colony-formation assay. TNFAIP8-silenced and control cells were seeded in a 6 -well plate with $\sim 500$ cells in $2 \mathrm{ml}$ complete culture medium/well. Cisplatin $(5 \mu \mathrm{g} / \mathrm{ml})$ was added to the medium after 2 days, and the cells were cultured at $37^{\circ} \mathrm{C}$ for $\sim 2$ weeks. The medium was discarded and the cells were washed gently with PBS when the clones were visible. Cells were fixed with $100 \%$ methanol for $15 \mathrm{~min}$ and stained with crystal violet for $20 \mathrm{~min}$ at room temperature. The clones were scanned and counted using Image-Pro Plus 6.0 (Media Cybernetics, Inc., Rockville, MD, USA).

MTS assay. Cell viability was measured with an MTS kit (G5421; Promega Corporation) according to the manufacturer's protocol. Cells were seeded at $2 \times 10^{3}$ cells/well in a 96-well plate and allowed to attach overnight. Different concentrations $(0,2.5,5,10,20$ or $40 \mu \mathrm{g} / \mathrm{ml})$ of cisplatin were added to the medium for $24 \mathrm{~h}$, or $5 \mu \mathrm{g} / \mathrm{ml}$ cisplatin was added to the medium for $6,12,24,48$ or $72 \mathrm{~h}$. Each group contained six well replicates. A total of $20 \mu \mathrm{l}$ MTS aqueous solution was added to each well and the cells were incubated at $37^{\circ} \mathrm{C}$ for $2 \mathrm{~h}$. Optical density values were measured at $490 \mathrm{~nm}$ on a full wavelength multi-function microplate reader (Thermo Fisher Scientific, Inc.).

Apoptosis analysis. Cells were seeded at $1 \times 10^{5}$ cells into each well of a 6-well plate with $2 \mathrm{ml}$ complete culture medium. The cells were left to attach overnight. Subsequently, cisplatin was added at a concentration of 5 or $10 \mu \mathrm{g} / \mathrm{ml}$ for $24 \mathrm{~h}$. Cells were collected and washed twice with DMEM, and apoptotic cells were stained with Annexin V-fluorescein isothiocyanate and propidium iodide (Miltenyi Biotec, Inc., Cambridge, MA, USA) according to the manufacturer's protocol. The cells were detected using a flow cytometer (FACSCalibur) and analyzed with FlowJo software (version 10; FlowJo LLC, Ashland, OR, USA).

Trypan blue staining. A total of $1 \times 10^{5}$ cells/well were seeded into a 6-well plate. Subsequent to cells attaching overnight, the medium was replaced with fresh medium with or without cisplatin (5 and $10 \mu \mathrm{g} / \mathrm{ml}$ ) and incubated for a further $24 \mathrm{~h}$. Cells were collected and the viability was determined by trypan blue staining at room temperature for $2 \mathrm{~min}$. Cell numbers were counted manually using a hemocytometer, and the cell death ratio was calculated as the proportion of trypan blue-positive cells to the total number of cells.

Western blot analysis. Cells were harvested, and total protein was extracted using an appropriate volume of lysis buffer (P0013; Beyotime Institute of Biotechnology). Supernatants were collected following centrifugation at $13,000 \mathrm{x} \mathrm{g}$ for $10 \mathrm{~min}$ at $4^{\circ} \mathrm{C}$. The protein concentration was determined using a bicinchoninic acid protein assay kit. Equal quantities of $40 \mu \mathrm{g}$ protein samples were loaded onto $10 \%$ gels for SDS-PAGE. Proteins were subsequently transferred to polyvinylidene fluoride membranes. Subsequent to blocking with 5\% skimmed milk at room temperature for $1 \mathrm{~h}$, the membranes were incubated with the following primary antibodies at $4{ }^{\circ} \mathrm{C}$ overnight: TNFAIP8 (1:1,000; ab195810; Abcam); $\beta$-actin (1:20,000; a1978; Sigma-Aldrich; Merck KGaA); procaspase-3 and cleaved caspase 3 (9662), cleaved caspase-8 (9496), phosphorylated-p38 (9211), p-38 (9212) and Bcl-2 (4223) at 
A
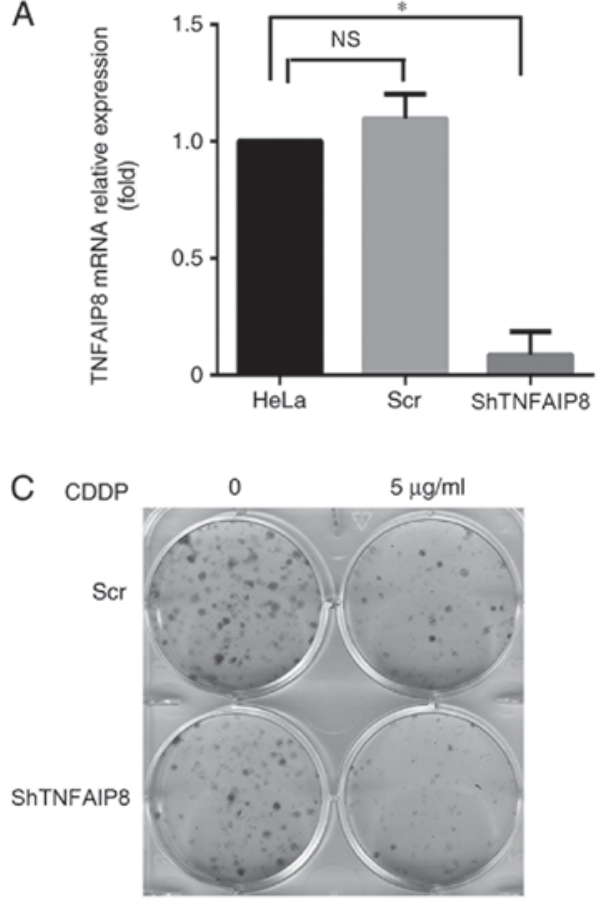

E

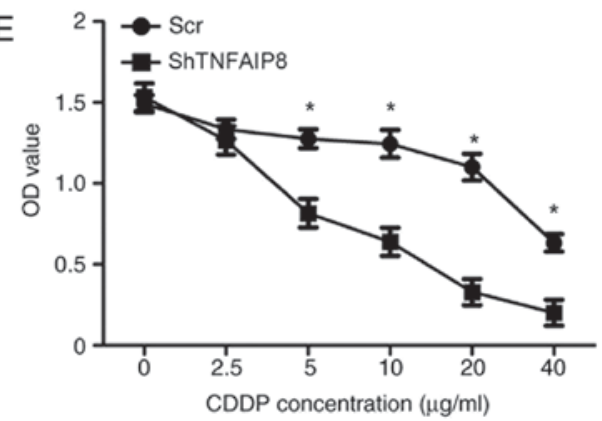

B
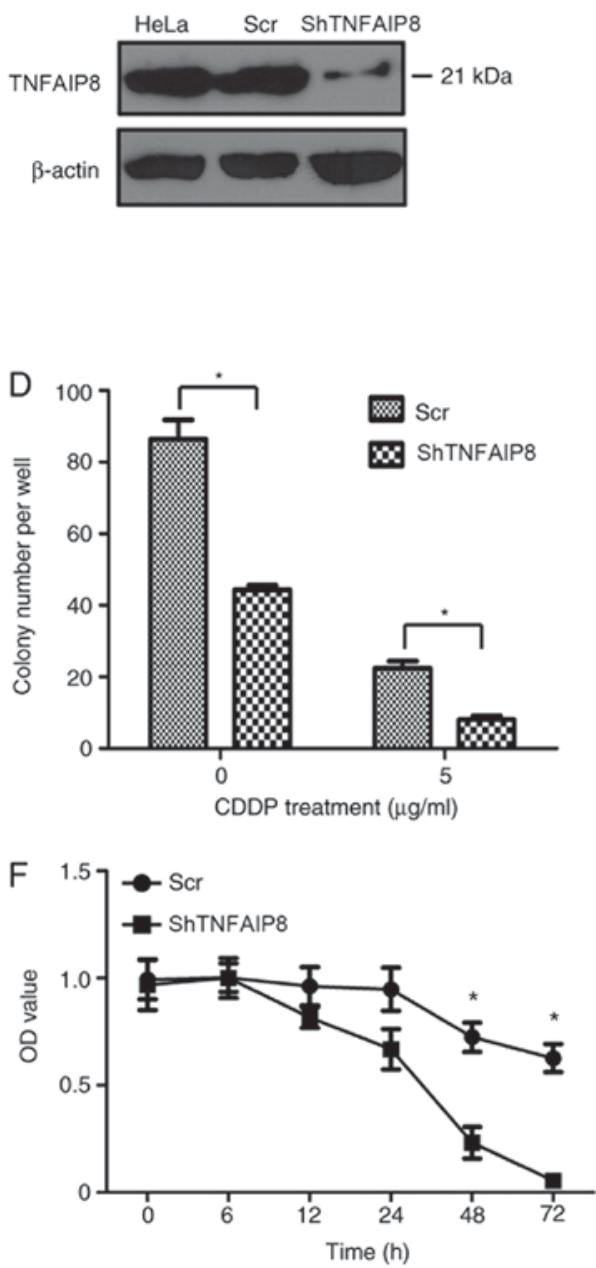

Figure 2. Depletion of TNFAIP8 impairs HeLa cell proliferation and viability. (A) TNFAIP8 mRNA relative expression in cells was detected using quantitative polymerase chain reaction. TNFAIP8 expression in HeLa cells was set as 1. (B) TNFAIP8 protein expression in cells was detected by western blotting. (C) A colony formation assay was performed with TNFAIP8-silenced HeLa cells and control cells. (D) Number of colonies formed by TNFAIP8-silenced HeLa cells and control cells in the presence or absence of CDDP. (E) Cellular viability of TNFAIP8-silenced HeLa cells and control cells treated with the stated concentrations of CDDP for $24 \mathrm{~h}$. (F) Cellular viability of TNFAIP8-silenced HeLa cells and control cells treated with $10 \mu \mathrm{g} / \mathrm{ml}$ cisplatin for the stated period of time. ${ }^{*} \mathrm{P}<0.05$. All the data are presented as the mean \pm standard deviation, with each of the experiments repeated in triplicate. TNFAIP8, tumor necrosis factor $\alpha$-induced protein 8 ; Scr, scrambled control RNA; shTNFAIP8, TNFAIP8-silencing short hairpin RNA; CDDP, cisplatin; OD, optical density; NS, not significant.

1:1,000 (Cell Signaling Technology, Inc., Danvers, MA, USA). Blot were then washed, and incubated with HRP-conjugated secondary antibody (1:500; BA1056; Wuhan Boster Biological Technology, Ltd.) at room temperature for $2 \mathrm{~h}$. Protein bands were visualized using an enhanced chemiluminescence system (Thermo Fisher Scientific, Inc.).

Statistical analysis. Data are presented as the mean \pm standard deviation of at least three independent experiments. The data was analyzed using a Student's t-test or one-way analysis of variance followed by Bonferroni correction using GraphPad Prism 6 (GraphPad Software, Inc., La Jolla, CA, USA). P<0.05 was considered to indicate a statistically significant difference.

\section{Results}

TNFAIP8 overexpression in cervical cancer tissues is associated with cisplatin resistance. A total of 40 pairs of cervical cancer tissues and non-tumor adjacent tissues were collected for immunohistochemical analysis of TNFAIP8 expression. As expected, TNFAIP8 was highly expressed in cervical cancer tissues, whereas, little or no staining was observed in the tissues adjacent to the tumor (Fig. 1A and B). The patients with high TNFAIP8 expression (18 cases) were significantly more likely to develop cisplatin resistance (16 cases of the total 22 cases) compared with patients with low TNFAIP8 expression (Fig. 1C; $\mathrm{P}<0.05)$. These data suggested that TNFAIP8 is an oncogene, which may mediate therapeutic resistance to cisplatin.

TNFAIP8 increases the proliferation of cervical cancer cells. To elucidate the function of TNFAIP8 in the growth of cervical cancer cells, HeLa cells were transfected with the plasmid pGLV-shTNFAIP8 or a control vector to generate TNFAIP8-knockdown and control HeLa cell lines (Fig. 2A and B). Equivalent numbers of each cell line were seeded into different wells of a flat plate to evaluate 
A

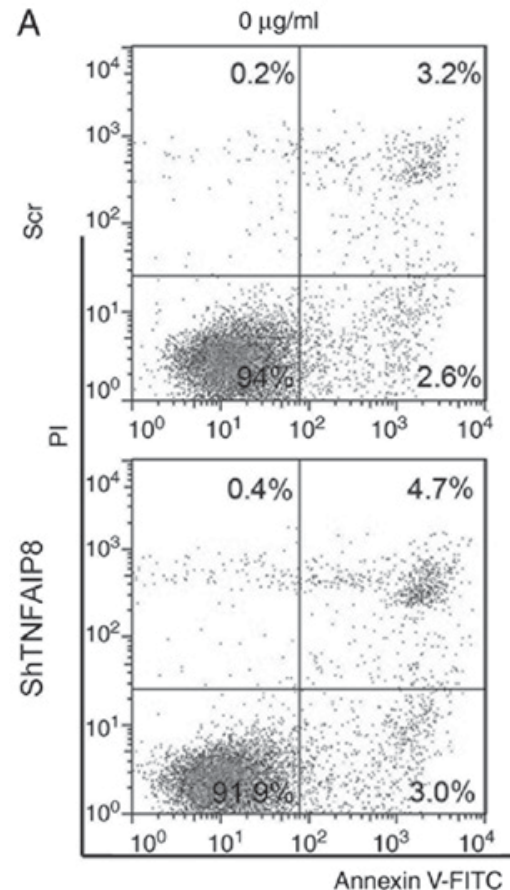

B

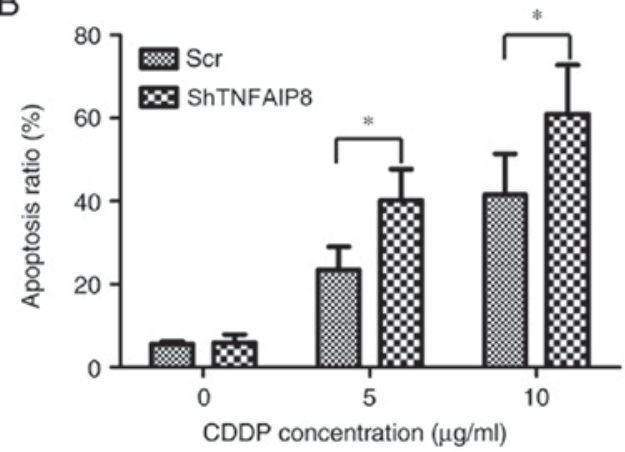

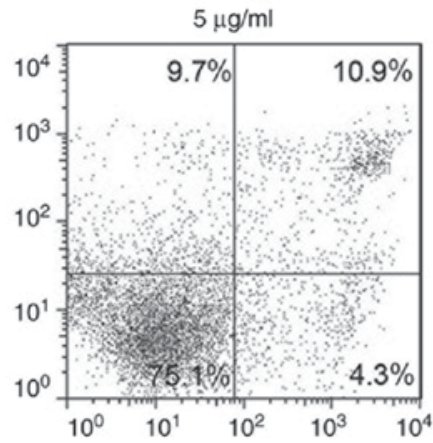
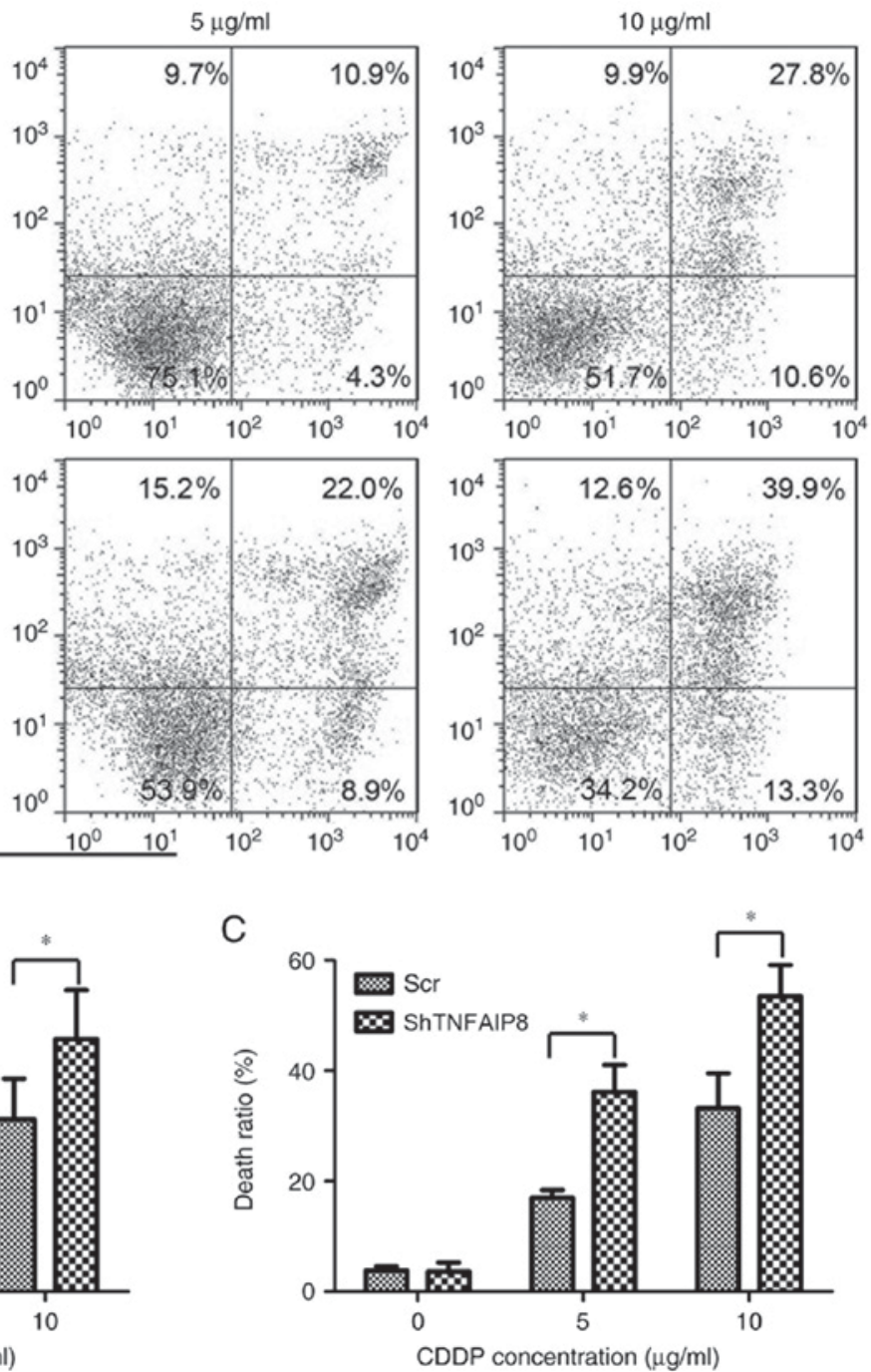

Figure 3. Depletion of TNFAIP8 promotes cellular apoptosis and death. (A) Cellular apoptosis of TNFAIP8-depleted HeLa and control cells subsequent to treatment with CDDP was evaluated using flow cytometry. (B) Cellular apoptotic ratio of HeLa cells with TNFAIP8 knockdown and control cells following treatment with CDDP was calculated, according to the flow cytometric analysis. (C) Cellular death ratio of TNFAIP8-depleted HeLa and control cells following treatment with CDDP was examined by trypan blue staining. ${ }^{*} \mathrm{P}<0.05$. Data are representative of three independent experiments. TNFAIP8, tumor necrosis factor $\alpha$-induced protein 8; Scr, scrambled control RNA; shTNFAIP8, TNFAIP8-silencing short hairpin RNA; FITC, fluorescein isothiocyanate; PI, propidium iodide; CDDP, cisplatin.

colony formation. After $\sim 2$ weeks, visible clones were stained with crystal violet, and the number of clones was counted and analyzed. A significant decrease in the proliferation rate of HeLa cells with decreased expression of TNFAIP8 was observed $(\mathrm{P}<0.05)$. In particular, fewer and smaller clones formed with TNFAIP8 knockdown cells compared with the control HeLa cells following treatment with cisplatin (Fig. 2C and D).

Knockdown of TNFAIP8 enhances the cytotoxicity of cisplatin in HeLa cells. To determine whether TNFAIP8 protein expression in cervical cancer was associated with cisplatin resistance, an MTS assay was used to measure cell viability. As approximately all cells in the shRNA group and control group died within $48 \mathrm{~h}$ following treatment with $10 \mathrm{mg} / \mathrm{ml}$ cisplatin (data not shown), cells were instead treated with $5 \mathrm{mg} / \mathrm{ml}$ cisplatin for $6,12,24,48$ or $72 \mathrm{~h}$. Only a small number of live HeLa cells were observed in the TNFAIP8 shRNA group, compared with the numerous cells that were still viable in the control group after cisplatin treatment for $72 \mathrm{~h}$ (Fig. 2F). Treatment with cisplatin significantly decreased the HeLa cell viability in a dose and time dependent manner as demonstrated in Fig. 2E and F, respectively.

Knockdown of TNFAIP8 promotes cellular apoptosis and death. The effect of TNFAIP8 knockdown on apoptosis and death in HeLa cells was examined. Following treatment with cisplatin at the stated concentrations for $24 \mathrm{~h}$, cellular apoptosis was detected using flow cytometry. The percentage of apoptotic HeLa cells in the TNFAIP8-knockdown group was significantly increased compared with the control group following treatment with cisplatin (Fig. $3 \mathrm{~A}$ and $\mathrm{B} ; \mathrm{P}<0.05$ ). Additionally, the level of cell death, as analyzed by trypan blue staining, demonstrated results similar to those for cellular apoptosis (Fig. 3C). Collectively, the present data demonstrated that silencing TNFAIP8 resulted in an increase in the cytotoxicity of cisplatin in HeLa cells, suggesting that TNFAIP8 confers resistance to cisplatin in cervical cancer. 
A

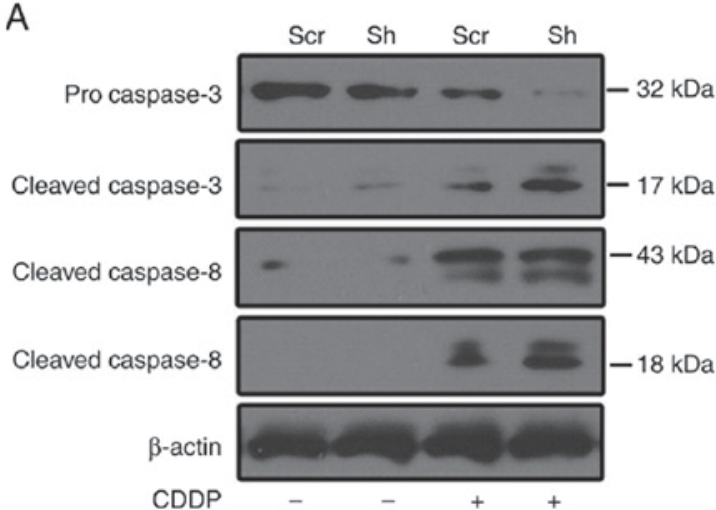

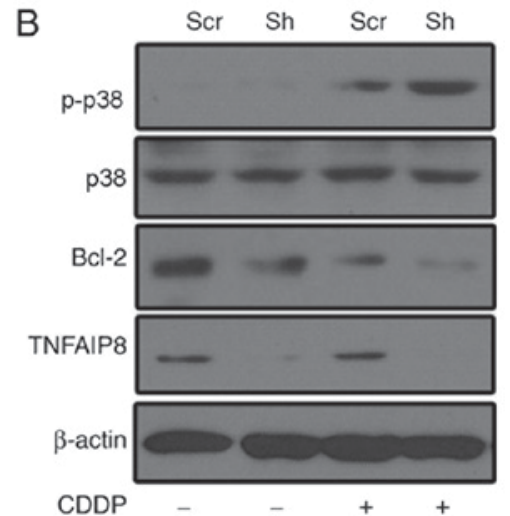

Figure 4. Depletion of TNFAIP8 affects cellular apoptosis and the p38 mitogen-activated protein kinase signaling pathway. (A) Caspase-3 and caspase-8 activation in control HeLa cells or in TNFAIP8-silenced cells, in the presence or absence of cisplatin (5 $\mu \mathrm{g} / \mathrm{ml}$ ). (B) Expression levels of p38, p-p38, Bcl-2 and TNFAIP8 in TNFAIP8-silenced HeLa and control cells in the presence or absence of CDDP (5 $\mu \mathrm{g} / \mathrm{ml})$. Data are representative of three independent experiments. TNFAIP8, tumor necrosis factor $\alpha$-induced protein 8; Scr, scrambled control RNA; CDDP, cisplatin; Bcl-2, B-cell lymphoma 2; p-, phosphorylated.

TNFAIP8 promotes cisplatin resistance by inhibiting cellular apoptosis. To investigate the underlying mechanisms of TNFAIP8 function in cisplatin resistance, caspase activation was evaluated following treatment with cisplatin. As presented in Fig. 4A, caspase-3 and -8 were activated subsequent to treatment with cisplatin, and TNFAIP8 silencing enhanced caspase activation in HeLa cells following treatment with cisplatin. Compared with the control group, Bcl-2 expression was decreased in the TNFAIP8-silenced group following treatment with cisplatin (Fig. 4B). To determine whether p38 mitogen-activated protein kinase (MAPK) contributes to cisplatin resistance in TNFAIP8-silenced HeLa cells, p38 MAPK activation was assessed. p38 signaling was activated in cisplatin-treated HeLa cells, and p38 phosphorylation was enhanced in the TNFAIP8-knockdown group subsequent to treatment with cisplatin (Fig. 4B). These data suggested that TNFAIP8 serves as an anti-apoptotic protein against cisplatin-induced cell death. This effect may aggravate chemotherapeutic treatment failure in patients with cervical cancer.

\section{Discussion}

To date, four members of the TNFAIP8 family of proteins have been characterized in mammals; TNFAIP8 (or TIPE), TNFAIP8-like 1 (TIPE1), TIPE2 and TIPE3 (22). These proteins possess different biological functions, although they possess high sequence consensus and share a death effecter domain (DED) (22). TNFAIP8 is the principal member of this family that is induced by TNF- $\alpha$ in response to environmental stress (11). It has previously been identified that TNFAIP8 is overexpressed in a wide range of different cancer types, and additional previous studies have demonstrated that it serves as an oncoprotein, as it induces the development and progression of tumors, and enhances cancer cell survival, proliferation and metastasis $(12,23,24)$. However, little is known regarding the biological and pathological functions of TNFAIP8 in cervical cancer. In the present study, a significant increase in TNFAIP8 expression was observed in cervical cancer tissues compared with the adjacent tissues. Furthermore, when TNFAIP8 was silenced in HeLa cells, cell colony formation and viability were impaired. This supported the hypothesis that TNFAIP8 serves as an oncogene, and promotes cervical cancer cell proliferation and survival.

Cisplatin is a widely used chemotherapeutic drug in the treatment of numerous types of solid tumors, including head and neck, ovarian, testicular, small-cell lung and cervical cancer (25). However, cisplatin is limited as an effective treatment due to dose-associated toxicities and adverse side-effects, whereas, the intrinsic or acquired resistance of cells to cisplatin inevitably causes treatment failure (26). Therefore, the two significant challenges associated with cisplatin therapy are identifying a safe and tolerable dose, and addressing the issue of cisplatin resistance. A previous study on the mechanisms of cisplatin resistance have demonstrated that tumor cells develop resistance as a natural self-defense mechanism to protect cells from the cytotoxic effect of cisplatin and to support cell survival (8). TNFAIP8 is a member of the Fas-associated death domain-like interleukin-1 $\beta$-converting enzyme caspase-8inhibitory protein family of anti-apoptotic proteins that contain a DED and as a result, may cause the inhibition of caspase-mediated cellular apoptosis $(9,10)$. Overexpression of TNFAIP8 in cancer cells is associated with enhanced survival and with inhibition of the apoptotic enzymes caspase- 8 and caspase-3 (27). In the present study, knockdown of TNFAIP8 expression in HeLa cells inhibited caspase- 8 and caspase- 3 activation, upon treatment with cisplatin, demonstrating that TNFAIP8 may be an anti-apoptotic protein that serves an important role in cellular survival and cisplatin resistance.

A previous study suggested that dysregulation of the anti-apoptotic protein Bcl-2 constitutes a risk factor for tumorigenesis (28). Additionally, the increased expression of Bcl-2 in a variety of cancer types has been associated with resistance to chemotherapeutic drugs, including cisplatin (29). In the present study, TNFAIP8 knockdown decreased Bcl-2 expression in cisplatin-treated cervical cancer cells. Along with the observation that depletion of TNFAIP8 improved cisplatin sensitivity and promoted cisplatin-induced cellular apoptosis, the present data suggested that TNFAIP8 is involved in cisplatin resistance by regulating Bcl-2 expression and suppressing cellular apoptosis.

p38 MAPKs are serine/threonine protein kinases that are involved in a variety of intracellular signaling events, and modulate varied biological effects, including proliferation, apoptosis, 
differentiation and senescence (30). In mammalian cells, four isoforms of p38 MAPK have been identified; however, the physiological and pathophysiological functions of the individual isoforms remain largely unknown. Despite high amino acid sequence similarity, the p38 MAPK isoforms present marked differences in tissue expression and functions. p38 $\alpha$ and p38 $\beta$ are ubiquitous, whereas, p38 $\gamma$ and $\mathrm{p} 38 \delta$ are tissue specific (31). The different $\mathrm{p} 38$ isoforms have been demonstrated to exert opposing effects. For example, p38 $\alpha$ suppresses inflammation-associated initiation of colon tumors; however, contributes to the proliferation and survival of colon tumor cells (32). In vascular endothelial cells, p38 $\alpha$ mediates pro-apoptotic signaling from transforming growth factor- $\beta 1$-induced apoptosis, whereas, $\mathrm{p} 38 \beta$ relays survival signals from pro-survival factors, including vascular endothelial growth factor (33). Given the duality of p38 MAPK functions in mammalian cells, the role of $\mathrm{p} 38$ MAPK was investigated in TNFAIP8-silenced HeLa cells. In the present study, it was demonstrated that treatment with cisplatin activated the p38 signaling pathway, and that TNFAIP8 silencing promoted p38 phosphorylation in cisplatin-treated HeLa cells. Additionally, the present data demonstrated that when TNFAIP8 was silenced, cisplatin-induced HeLa cell apoptosis and death were enhanced. Therefore, it was suggested that p38 MAPK activation likely serves a negative role in TNFAIP8-mediated cisplatin resistance, and that regulating p38 MAPK activity may be used to reverse cisplatin resistance in women with cervical cancer.

Numerous proteins, including cluster of differentiation (CD)44, CD24, CD38, Fos-related antigen-1, heat shock protein $\beta-1$ and transcription factor SOX-9, have been implicated in cervical cancer, and dysregulation of these proteins is closely associated with the occurrence and outcome of cervical cancer (34-39). However, the etiology of cervical carcinoma remains elusive. In the present study, it was demonstrated that in cervical cancer cells, TNFAIP8 overexpression is associated with resistance to cisplatin, whereas, TNFAIP8 silencing improved cisplatin sensitivity in HeLa cells. The present data suggested that TNFAIP8 may be considered a promising therapeutic target for the treatment of cervical cancer.

\section{Acknowledgements}

The authors would like to thank Professor Jiang Wu and Mrs Xi Chen from the Pathology Department of Henan University Huaihe Hospital for their technical assistance.

\section{Funding}

No funding was received.

\section{Availability of data and materials}

The datasets used and analyzed during the present study are available from the corresponding author on reasonable request.

\section{Authors' contributions}

SW and LC conceived the study and wrote the article. SW and WL designed and performed the experiments, and analyzed the data. ZW, TC, PW, NL, XL and MC were involved in the design or execution of a number of experiments. SZ and YL designed and performed the human tumor studies. YM made suggestions for the design of the study and contributed to revision of the manuscript and approved the version to be submitted. All authors have read and approved the final manuscript.

\section{Ethics approval and consent to participate}

The present study complies with current ethical considerations and the protocol was approved by the Committee for Ethical Review at Henan University School of Medicine (Kaifeng, China). Written informed consent was provided by all patients.

\section{Patient consent for publication}

Not applicable.

\section{Competing interests}

The authors declare that they have no competing interests.

\section{References}

1. Jemal A, Bray F, Center MM, Ferlay J, Ward E and Forman D: Global cancer statistics. CA Cancer J Clin 61: 69-90, 2011.

2. Parish SL, Swaine JG, Son E and Luken K: Determinants of cervical cancer screening among women with intellectual disabilities: Evidence from medical records. Public Health Rep 128: 519-526, 2013.

3. Diaz-Padilla I, Monk BJ, Mackay HJ and Oaknin A: Treatment of metastatic cervical cancer: Future directions involving targeted agents. Crit Rev Oncol Hematol 85: 303-314, 2013.

4. Takekuma M, Kuji S, Tanaka A, Takahashi N, Abe M and Hirashima Y: Platinum sensitivity and non-cross-resistance of cisplatin analogue with cisplatin in recurrent cervical cancer. J Gynecol Oncol 26: 185-192, 2015.

5. Zhu M, Zhou X, Du Y, Huang Z, Zhu J, Xu J, Cheng G, Shu Y, Liu $\mathrm{P}$, Zhu W, et al: miR-20a induces cisplatin resistance of a human gastric cancer cell line via targeting CYLD. Mol Med Rep 14: 1742-1750, 2016.

6. Mehta FF, Baik S and Chung SH: Recurrence of cervical cancer and its resistance to progestin therapy in a mouse model. Oncotarget 8: 2372-2380, 2017.

7. Bi L, Ma F, Tian R, Zhou Y, Lan W, Song Q and Cheng X: AJUBA increases the cisplatin resistance through hippo pathway in cervical cancer. Gene 644: 148-154, 2018.

8. Shen DW, Pouliot LM, Hall MD and Gottesman MM: Cisplatin resistance: A cellular self-defense mechanism resulting from multiple epigenetic and genetic changes. Pharmacol Rev 64: 706-721, 2012.

9. Kumar D, Whiteside TL and Kasid U: Identification of a novel tumor necrosis factor-alpha-inducible gene, SCC-S2, containing the consensus sequence of a death effector domain of fas-associated death domain-like interleukin-1beta-converting enzyme-inhibitory protein. J Biol Chem 275: 2973-2978, 2000.

10. Kumar D, Gokhale P, Broustas C, Chakravarty D, Ahmad I and Kasid U: Expression of SCC-S2, an antiapoptotic molecule, correlates with enhanced proliferation and tumorigenicity of MDA-MB 435 cells. Oncogene 23: 612-616, 2004.

11. Patel S, Wang FH, Whiteside TL and Kasid U: Identification of seven differentially displayed transcripts in human primary and matched metastatic head and neck squamous cell carcinoma cell lines: Implications in metastasis and/or radiation response. Oral Oncol 33: 197-203, 1997.

12. Goldsmith JR and Chen YH: Regulation of inflammation and tumorigenesis by the TIPE family of phospholipid transfer proteins. Cell Mol Immunol 14: 1026, 2017.

13. Zhang LJ, Liu X, Gafken PR, Kioussi C and Leid M: A chicken ovalbumin upstream promoter transcription factor I (COUP-TFI) complex represses expression of the gene encoding tumor necrosis factor alpha-induced protein 8 (TNFAIP8). J Biol Chem 284: 6156-6168, 2009. 
14. Xiao M, Xu Q, Lou C, Qin Y, Ning X, Liu T, Zhao X, Jia S and Huang Y: Overexpression of TNFAIP8 is associated with tumor aggressiveness and poor prognosis in patients with invasive ductal breast carcinoma. Hum Pathol 62: 40-49, 2017.

15. Sun Z, Liu X, Song JH, Cheng Y, Liu Y, Jia Y, Meltzer SJ and Wang Z: TNFAIP8 overexpression: A potential predictor of lymphatic metastatic recurrence in pN0 esophageal squamous cell carcinoma after Ivor Lewis esophagectomy. Tumour Biol 37: 10923-10934, 2016

16. Li Y, Jing C, Chen Y, Wang J, Zhou M, Liu X, Sun D, Mu L, $\mathrm{Li} \mathrm{L}$ and Guo X: Expression of tumor necrosis factor $\alpha$-induced protein 8 is upregulated in human gastric cancer and regulates cell proliferation, invasion and migration. Mol Med Rep 12: 2636-2642, 2015.

17. Yang M, Zhao Q, Wang X, Liu T, Yao G, Lou C and Zhang Y: TNFAIP8 overexpression is associated with lymph node metastasis and poor prognosis in intestinal-type gastric adenocarcinoma. Histopathology 65: 517-526, 2014.

18. Liu T, Gao H, Yang M, Zhao T, Liu Y and Lou G: Correlation of TNFAIP8 overexpression with the proliferation, metastasis, and disease-free survival in endometrial cancer. Tumour Biol 35: 5805-5814, 2014.

19. Zhang C, Chakravarty D, Sakabe I, Mewani RR, Boudreau HE, Kumar D, Ahmad I and Kasid UN: Role of SCC-S2 in experimental metastasis and modulation of VEGFR-2, MMP-1, and MMP-9 expression. Mol Ther 13: 947-955, 2006.

20. Gus-Brautbar Y, Johnson D, Zhang L, Sun H, Wang P, Zhang S, Zhang L and Chen YH: The anti-inflammatory TIPE2 is an inhibitor of the oncogenic Ras. Mol Cell 45: 610-618, 2012.

21. Livak KJ and Schmittgen TD: Analysis of relative gene expression data using real-time quantitative PCR and the $2^{-\Delta \Delta C_{\mathrm{T}}}$ method. Methods 25: 402-408, 2001.

22. Sun H, Gong S, Carmody RJ, Hilliard A, Li L, Sun J, Kong L, $\mathrm{Xu} \mathrm{L}$, Hilliard $\mathrm{B}, \mathrm{Hu} \mathrm{S}$, et al: TIPE2, a negative regulator of innate and adaptive immunity that maintains immune homeostasis. Cell 133: 415-426, 2008.

23. Lou Y and Liu S: The TIPE (TNFAIP8) family in inflammation, immunity, and cancer. Mol Immunol 49: 4-7, 2011.

24. Fayngerts SA, Wu J, Oxley CL, Liu X, Vourekas A, Cathopoulis T, Wang Z, Cui J, Liu S, Sun H, et al: TIPE3 is the transfer protein of lipid second messengers that promote cancer. Cancer Cell 26 : 465-478, 2014

25. Galluzzi L, Senovilla L, Vitale I, Michels J, Martins I, Kepp O, Castedo M and Kroemer G: Molecular mechanisms of cisplatin resistance. Oncogene 31: 1869-1883, 2012.

26. Siddik ZH: Cisplatin: Mode of cytotoxic action and molecular basis of resistance. Oncogene 22: 7265-7279, 2003.

27. You Z, Ouyang H, Lopatin D, Polver PJ and Wang CY: Nuclear factor-kappa B-inducible death effector domain-containing protein suppresses tumor necrosis factor-mediated apoptosis by inhibiting caspase-8 activity. J Biol Chem 276: 26398-26404, 2001.
28. Yang TM, Barbone D, Fennell DA and Broaddus VC: Bcl-2 family proteins contribute to apoptotic resistance in lung cancer multicellular spheroids. Am J Respir Cell Mol Biol 41: 14-23, 2009.

29. Low SY, Tan BS, Choo HL, Tiong KH, Khoo AS and Leong CO: Suppression of BCL-2 synergizes cisplatin sensitivity in nasopharyngeal carcinoma cells. Cancer Lett 314: 166-175, 2012.

30. Chang L and Karin M: Mammalian MAP kinase signalling cascades. Nature 410: 37-40, 2001

31. Cuadrado A and Nebreda AR: Mechanisms and functions of p38 MAPK signalling. Biochem J 429: 403-417, 2010.

32. Gupta J, del Barco Barrantes I, Igea A, Sakellariou S, Pateras IS, Gorgoulis VG and Nebreda AR: Dual function of p38 $\alpha$ MAPK in colon cancer: Suppression of colitis-associated tumor initiation but requirement for cancer cell survival. Cancer Cell 25: 484-500, 2014.

33. Ferrari G, Terushkin V, Wolff MJ, Zhang X, Valacca C, Poggio P, Pintucci G and Mignatti P: TGF- $\beta 1$ induces endothelial cell apoptosis by shifting VEGF activation of $\mathrm{p} 38^{\mathrm{MAPK}}$ from the prosurvival p38 $\beta$ to proapoptotic p38 $\alpha$. Mol Cancer Res 10: 605-614, 2012.

34. Xiao S, Zhou Y, Yi W, Luo G, Jiang B, Tian Q, Li Y and Xue M: Fra-1 is downregulated in cervical cancer tissues and promotes cervical cancer cell apoptosis by p53 signaling pathway in vitro. Int J Oncol 46: 1677-1684, 2015.

35. Wobus M, Kuns R, Wolf C, Horn LC, Köhler U, Sheyn I, Werness BA and Sherman LS: CD44 mediates constitutive type I receptor signaling in cervical carcinoma cells. Gynecol Oncol 83: 227-234, 2001.

36. Liao S, Xiao S, Zhu G, Zheng D, He J, Pei Z, Li G and Zhou Y: CD38 is highly expressed and affects the PI3K/Akt signaling pathway in cervical cancer. Oncol Rep 32: 2703-2709, 2014

37. Wu JH, Liang XA, Wu YM, Li FS and Dai YM: Identification of DNA methylation of SOX9 in cervical cancer using methylated-CpG island recovery assay. Oncol Rep 29: 125-132, 2013.

38. Dobo C, Stavale JN, Lima Fde O, Ribeiro DA, Arias V, Gomes TS and Oshima CT: HSP27 is commonly expressed in cervical intraepithelial lesions of Brazilian women. Asian Pac J Cancer Prev 14: 5007-5010, 2013

39. Pei Z, Zhu G, Huo X, Gao L, Liao S, He J, Long Y, Yi H, Xiao S, Yi W, et al: $\mathrm{CD} 24$ promotes the proliferation and inhibits the apoptosis of cervical cancer cells in vitro. Oncol Rep 35: 1593-1601, 2016 